\title{
Volumen maderable de mangle negro (Avicennia germinans L.) impactado por herbivoria de Anacamptodes sp en Cárodenas Tabasco
}

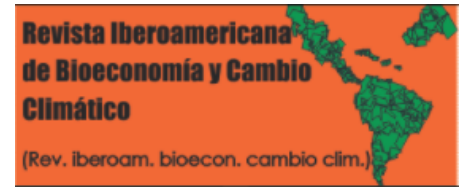

\author{
Timber volume of black mangrove (Avicennia germinans L.) \\ impacted by Anacamptodes sp herbivory in Cárdenas Tabasco
}

\author{
Sol-Sánchez, Ángel; Sánchez-Gutiérrez, Facundo; Hernández-Melcho, \\ Gloria Isela; Zamora Cornelio, Luis Felipe; Sardiñas Gómez, Oreste; \\ Rivera, Carlos; Toruño, Pedro José; Editor Académico Prof. Dr. Carlos \\ A. Zúniga-González
}

Ángel Sol-Sánchez

sol@colpos.mx

Colegio de Postgraduados-Campus Tabasco., Mexico

(D) Facundo Sánchez-Gutiérrez

paco379@hotmail.com

Universidad Autónoma de Chiapas, Mexico

Gloria Isela Hernández-Melcho

isela7827@gmail.com

Colegio de Postgraduados-Campus Tabasco., Mexico

Luis Felipe Zamora Cornelio

tguayacan@hotmail.com

Ecología para la Conservación y el Desarrollo del

Trópico, A. C. , Mexico

Oreste Sardiñas Gómez

Instituto de Geografía Tropical, Cuba, Cuba

Carlos Rivera

crivera20@gmail.com

CURLA- UNAH, Honduras, Honduras

(iD) Pedro José Toruño

pjoseto@gmail.com

Universidad Nacional Autónoma de Nicaragua, León.,

Nicaragua

Editor Académico Prof. Dr. Carlos A. Zúniga-

González

Universidad Nacional Autónoma de Nicaragua, León,

Nicaragua

Revista Iberoamericana de Bioeconomía y Cambio Climático

Universidad Nacional Autónoma de Nicaragua, León, Nicaragua ISSN-e: 2410-7980

Periodicidad: Semestral

vol. 1 , núm. 1,2015

czuniga@ct.unanleon.edu.ni

Recepción: 12 Agosto 2014
Resumen: El presente trabajo se realizó en el ejido las coloradas, Cárdenas, Tabasco, con el objetivo de cuantificar el volumen maderable del mangle negro (Avicennia germinans (L.) L., afectado y muerto por la oruga de Anacamptodes sp., durante 2010-2011. Se establecieron 10 unidades de muestreo (UM) de forma rectangular de 20 x $50 \mathrm{~m}$ para evaluación permanente. Se registró el estado sanitario de los árboles, el diámetro a la altura del pecho (DAP $13 \mathrm{~m}$ ), la altura total y altura del fuste limpio (Ht y HA). Se calculó el área basal ( $\mathrm{AB}$ m.ha $\left.{ }^{-1}\right)$, Volumen total y del fuste limpio ( Vt y Vfl m.ha ${ }^{-1}$ ) y se estimó la densidad de árboles por especie. Los valores obtenidos se arreglaron en clases diamétricas con una amplitud de $5 \mathrm{~cm}$. Se realizó un análisis de correlación lineal simple entre la altura y el DAP. En total se evaluaron 1,831 árboles de tres especies, tres géneros y tres familias botánicas. El mangle negro representó $99.6 \%$ del total de árboles evaluados; la densidad varió de 750 hasta 2,520 árboles por ha ${ }^{-1}$. El 42.9\% de los árboles registrados mostraban partes vivas y el $57.1 \%$ estaban totalmente muertos. Específicamente, el mangle negro registró un Vt de $68.3 \mathrm{~m}$. ha ${ }^{-1}$, Vfl de $24.6 \mathrm{~m}$. ha ${ }^{-1} \mathrm{y}$ un $\mathrm{AB}$ de $12.4 \mathrm{~m}$. ha ${ }^{-1}$. La categoría de $5-9.9 \mathrm{~cm}$ de DAP registró el $45 \%$ de los árboles identificados. La correlación del DAP y la altura del mangle negro, fue moderada positiva significativa $\left(\mathrm{r}^{2}=\right.$ $\left.0.5313, \mathrm{p}=2.2 \mathrm{e}^{-16}\right)$.

Palabras clave: Volumen maderable, Anacamptodes, Mangle negro, herbivoría.

Abstract: The present work was carried out in the ejido las coloradas, Cárdenas, Tabasco, with the objective of quantifying the timber volume of the black mangrove (Avicennia germinans (L.) L., affected and killed by the caterpillar of Anacamptodes sp., during 2010-2011. 10 rectangular sampling units (MU) of $20 \times 50 \mathrm{~m}$ were established for permanent evaluation. The health status of the trees, the diameter at breast height (DBH $13 \mathrm{~m})$, the total height and height of the clean stem ( $\mathrm{Ht}$ and $\mathrm{HA})$. The basal area $\left(\mathrm{AB} \mathrm{m}{ }^{2} \mathrm{ha}^{-1}\right)$, total volume and of the clean stem ( $\mathrm{Vt}$ and Vfl $\mathrm{m}^{3} \mathrm{ha}^{-1}$ ) were calculated and the density of trees by species was estimated.The values obtained were arranged in diameter classes 
Aprobación: 12 Noviembre 2014

URL: http://portal.amelica.org/ameli/journal/394/3941748008/

DOI: https://doi.org/10.5377/ribcc.v1i1.2145

Autor de correspondencia: isela7827@gmail.com with an amplitude of $5 \mathrm{~cm}$. A simple linear correlation analysis was carried out between height and DBH. A total of 1,831 trees of three species, three genera and three botanical families were evaluated. The black mangrove represented $99.6 \%$ of the total of trees evaluated, the density varied from 750 to 2,520 trees. boles per ha-1. $42.9 \%$ of the registered trees showed live parts and $57.1 \%$ were totally dead. Specifically, the black mangrove recorded a Vt of $68.3 \mathrm{~m}^{3} \mathrm{ha}^{-1}$, Vfl of $24.6 \mathrm{~m}^{3} \mathrm{ha}^{-1}$, and an $\mathrm{AB}$ of $12.4 \mathrm{~m}^{2} \mathrm{ha}^{-1}$. The $5-9.9 \mathrm{~cm} \mathrm{DBH}$ category registered $45 \%$ of the identified trees. The correlation of $\mathrm{DBH}$ and the height of the black mangrove was moderate positive significant $\left(r^{2}=0.5313\right.$, $\mathrm{p}=2.2 \mathrm{e}-16)$.

Keywords: Timber volume, Anacamptodes, black mangrove, hervibory.

\section{INTRODUCCIÓN}

A nivel mundial los manglares representan el $1 \%$ de los bosques tropicales con 16 millones de ha (Flores et al., 2007). Estos son comunidades vegetales que existen a lo largo de las costas tropicales y subtropicales del mundo (Tovilla et al., 2004). Regularmente son inundados por las mareas con aguas marinas o estuarinas (salobres), sin o con poca influencia del oleaje, por lo que se desarrollan alrededor de las lagunas costeras, esteros y desembocadura de ríos y arroyos (INE y SEMARNAT, 2005; Castillo et al., 2010; Díaz, 2011). El sistema radical de algunas especies presenta raíces zancas y neumatóforos, que cumplen la función de sostén en el fondo lodoso y respiración radical (Díaz, 2011).

En el mundo, la diversidad de los mangles comprende alrededor de 69 especies y 12 géneros agrupados en 12 familias botánicas. En América se registran 10 especies de mangle; en México las más comunes son mangle rojo (Rhizophora mangle L.), mangle blanco (Laguncularia racemosa (L.) Gaerth), mangle negro (Avicennia germinans L.) y mangle botoncillo (Conocarpus erectus (Moreno et al., 2002; Hernández et al., 2008; Rico, 1982), las cuales están sujetas a protección especial de acuerdo a la NOM-059-SEMARNAT-2010. Estas especies están sujetas a herbivoría por diversas especies de vertebrados e invertebrados que aunque son elementos importantes de los ecosistemas, las condiciones ambientales puedes llevarlos a constituirse como plagas (Sol et al., 2012)

Los manglares son los ecosistemas más productivos del planeta generan $24 \mathrm{t}$ ha-1año de biomasa, en comparación con algunos cultivos como la caña de azúcar que produce $20 \mathrm{t}$ ha-1 año y los pastizales con 3 t ha-1 año (Flores et al., 2003; Valdez, 2004; Fonseca et al., 2007; Berlanga y Ruiz, 2007). En ellos habitan una gran diversidad de flora y fauna silvestre empleada para alimento, como son los peces y crustáceos, que inician su ciclo de vida dentro del manglar, aunque finalizan en mar abierto. Del mismo modo esos ambientes se constituyen como sitios de crianza y desarrollo de diversas especies, que aunque no tienen valor comercial constituyen parte importante de la cadena alimenticia del ecosistema (Valdez, 2004). Por otro lado se tienen recursos forestales maderables y no maderables como leña, madera, postes, carbón, taninos, miel que son muy importantes para la población local pues constituyen sus únicos recursos disponibles (INE y SEMARNAT, 2005; Valdez, 2004); Además generan bienes y servicios ambientales como el control de la erosión costera, estabilización de la línea de costa, captura de carbono, generación de oxígeno, protección y seguridad de las

\section{NotAS DE AUTOR}


costas ante el impacto de huracanes, y tsunamis y protección contra la brisa marina (INE y SEMARNAT, 2005; Tovilla et al., 2009).

A pesar de los múltiples beneficios ecológicos, económicos y culturales que los bosques de mangle ofrecen, son los ambientes tropicales costeros más amenazados, principalmente por la deforestación para el desarrollo portuario, petrolero, turístico, agropecuario, acuícola, asentamientos humanos y explotación forestal (INE y SEMARNAT, 2005; Berlanga y Ruiz, 2007; CONABIO, 2008).

Esta deforestación impacta negativamente a las pesquerías locales y de alta mar; por cada hectárea de manglar destruido, hay una pérdida de $800 \mathrm{~kg}$ anual de camarón y pescado de valor comercial sin considerar otros beneficios directos (Melchor Hernández et al., 2013). Considerando que las tasas de pérdida anual del manglar oscilan entre el 1 y el $2.5 \%$, para el año 2025 podría haberse perdido entre el 40 y el $50 \%$ de la superficie de manglar reportada para México en el año 2000 (INE y SEMARNAT, 2005).

México se ubica en el cuarto lugar mundial en relación a la superficie de manglar, con 770,057 ha, junto con Brasil, Colombia e Indonesia (Flores et al., 2003; Hernández et al., 2008). Estos manglares se distribuyen en los litorales del Océano Pacífico y Golfo de California (Chiapas hasta Sonora), y en el Golfo de México (Tovilla et al., 2009; Castillo et al., 2010).

Asimismo, en la república mexicana se registran manglares en 17 estados costeros, siendo Campeche, Yucatán y Sinaloa los que presentan mayores superficies con mas 196552 ha, 80528 ha y 71225 ha, respectivamente y que en suma representan el 53\% de la superficie nacional (CONABIO, 2008; Díaz, 2011).

Tabasco ocupa el séptimo lugar a nivel nacional con 35191 ha (5.4\%). Los municipios con mayor superficie son: Paraíso, Cárdenas, Centla, Jalpa de Méndez y Comalcalco, todos cuentan con las tres especies principales y algunas incluyen al mangle botoncillo (CONABIO, 2008).

Sin embargo, al igual que ocurre en el mundo y en otros estados de la República Mexicana, en Tabasco el manglar está sujeto a perturbaciones antrópicas principalmente por las actividades de la industria petrolera (Gallegos y Botello, 1988), que contaminan los suelos con derrames de petróleo (García et al., 2006); causando asfixia y muerte en plántulas y árboles (Olguín, 2007).

Aunado a lo anterior, en el 2010 la superficie del mangle negro se vio amenazada por la presencia desmesurada de orugas herbivoras de una polilla del genero Anacamptodes. Su presencia fue detectada en mayo del 2010 en el ejido Sinaloa como un brote aislado y único, misma que se disperso a lo largo del manglar afectando solo mangle negro (Avicennia germinans).

La severidad del daño fue de magnitud tal que los arboles colonizados por estas orugas fueron defoliados en su totalidad. En octubre del mismo año la CONAFOR tuvo conocimiento del problema pero desconocía su magnitud y para marzo del la última generación de orugas ya era adulta y había desaparecido del lugar. Después de esta etapa se inicio el reconocimiento de los daños, inicialmente a pie en las diversas comunidades impactadas y el saldo sumaba 3000 hectáreas, mismas que fueron corroboradas por vuelo aéreo y por restitución en mapas y el saldo total fue de 3,841 hectáreas defoliadas (vuelo aéreo CONAFOR Junio, 2011).

Para evaluar el daño se establecieron parcelas de monitoreo en campo en una de las comunidades afectadas y se obtuvo que el daño fue de tipo 2 ( Sol et al., 2002). Al respecto, no se tienen datos cuantitativos que permitan estimar el porcentaje de la defoliación del manglar para determinar si es posible su regeneración natural, en tal sentido, el presente estudio tuvo como objetivo determinar y cuantificar el volumen maderable de mangle negro (Avicennia germinans L.), que fue impactado por la oruga de Anacamptodes sp, en el ejido las Coloradas en Cárdenas, Tabasco, México.

\section{MATERIALES Y MÉTODOS}

El estudio se realizó en un área de manglar, ubicado en el Ejido las Coloradas, ampliación las Aldeas, entre las coordenadas geográficas $18^{\circ} 19^{\prime} 48.55^{\prime \prime} \mathrm{N}$ y 93³3'3.59"O, del municipio de Cárdenas, Tabasco (Figura 1). 


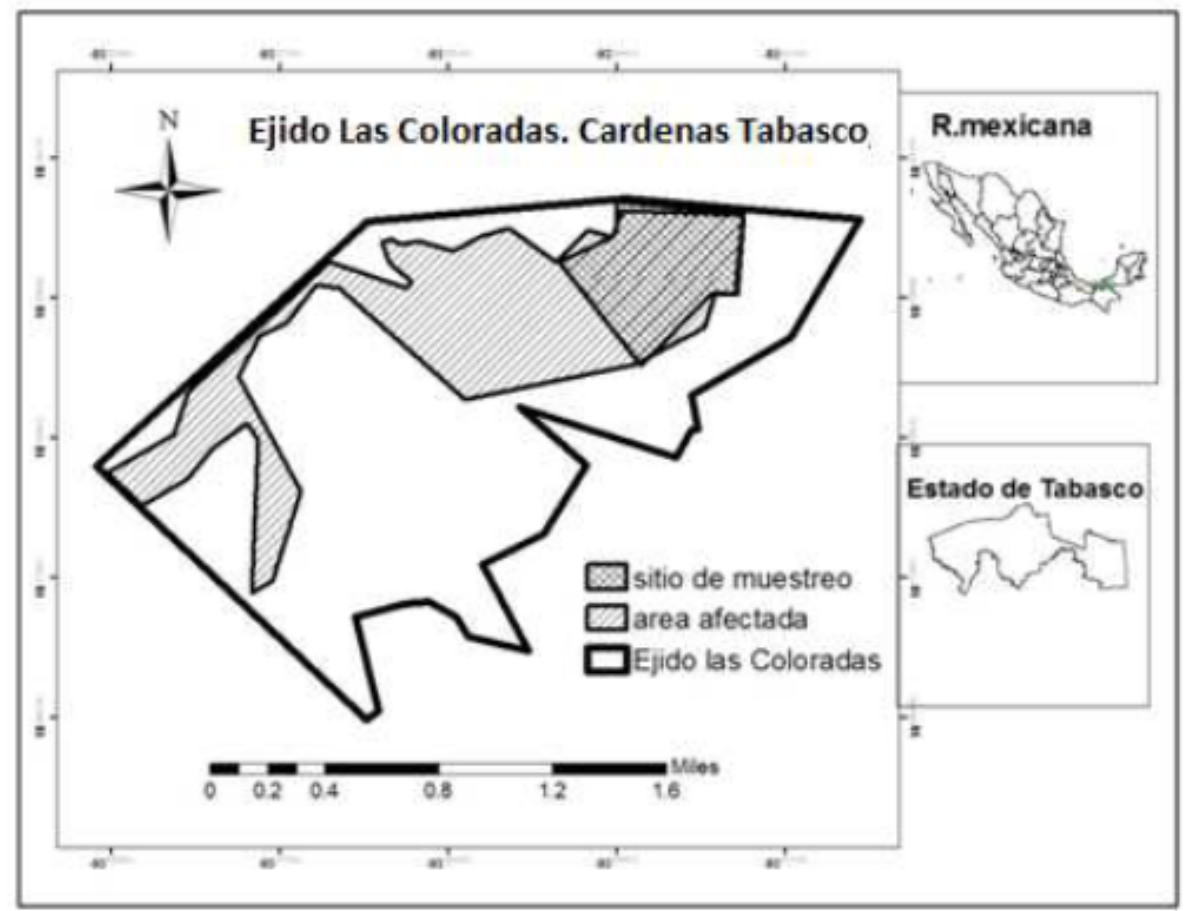

FIGURA 1

Ubicación geográfica del Ejido Las Coloradas, Cárdenas, Tabasco, México

Elaboración propia

\section{Diseño y procedimiento de muestreo}

Se establecieron 10 unidades de muestreo (UM) de forma rectangular de $20 \times 50 \mathrm{~m}(1,000 \mathrm{~m} 2)$ en transectos perpendiculares a la línea de costa. Cada cuadrante se delimitó con hilo rafia y se inventarío y marco cada uno de los árboles con pintura aerosol rojo. Se georeferenciaron los vértices con GPS, se identificaron taxonómicamente las especies arbóreas presentes, y su estado fitosanitario (Vivo o muerto) (Valdés, 2004).

Medición dasométrica y análisis estadístico

Se midió el diámetro a la altura del pecho (DAP) con una cinta diamétrica. Para el caso del mangle rojo (Rhizophora mangle L.) se midió $30 \mathrm{~cm}$ arriba de la última raíz aérea. La altura total y fuste limpio (Ht y Hf) se midieron con una Pistola Haga. Con base a estas variables se estimó, el área basal $(\mathrm{AB})$ con la ecuación:

$\mathrm{AB}=0.7854 \times \mathrm{D} 2$, donde $\mathrm{D}=$ diámetro $(\mathrm{m} 2 \mathrm{ha}-1), 0.7854$ = constante; el volumen total y comercial $(\mathrm{Vt}$, $\mathrm{Vfl}$ ) con la ecuación: $\mathrm{V}=\mathrm{AB} \times \mathrm{ff} \times \mathrm{H}$, donde $\mathrm{V}=$ volumen $\mathrm{m} 3$; $\mathrm{ff}=0.70$ y $\mathrm{H}=$ altura (Valdés, 2004; Valdés, 2002; Góngora, 2005; Tovilla et al., 2009).

La información del DAP de los individuos censados se organizó por categoría diamétrica a intervalos de $5 \mathrm{~cm}, 0.1$ a 4.9, 5 a 9.9, 10 a 14.9, y así sucesivamente, para conocer la representatividad de cada clase diamétrica con respecto al total (Ammour et al., 1999; Andenmatten y Letourneau, 2000). Se estimó la densidad promedio de los árboles por hectárea de acuerdo a los datos obtenidos en las unidades de monitoreo (Góngora et al., 2004; Tovilla y Orihuela, 2004).

Se realizó un análisis de correlación lineal simple con el paquete estadístico R commander entre la altura y el DAP del mangle negro en el ecosistema manglar con el fin de identificar posibles relaciones (Sol-Sánchez, 2012).

\section{Resultados}

Composición floristica y densidad de arboles 
En total se contabilizaron 1,831 árboles de tres especies, tres géneros y tres familias botánicas. Las especies registradas fueron mangle negro (A. germinans) con 1,823 árboles (99.6\%), mangle rojo $(R$. mangle) seis árboles $(0.3 \%)$ y mangle blanco $(L$. racemosa) dos árboles $(0.1 \%)$.

La densidad promedio por sitio de monitoreo fue de 183 árboles; variando de 75 a 252 árboles, con un promedio de 1,831 árboles ha-1; variando de 750 a 2,520 árboles ha ${ }^{-1}$. Se registraron 786 árboles vivos o parcialmente vivos y 1,045 muertos ( 42.9 y $57.1 \%$; respectivamente). La especie con mayor representatividad fue el mangle negro con una densidad de 1,823 árboles ha-1, de los cuales 783 estaban vivos al momento del muestreo (43\%) y 1,040 eran árboles muertos (57\%).

La densidad de árboles fue mayor en áreas con presencia de juveniles-, mientras que en sitios con árboles adultos ésta fue menor debido a la gran cobertura de las copas. En estos sitios, no se detectó evidencia de la presencia de la oruga. La densidad de árboles por hectárea y el diámetro de las mismas indican que el sitio se encontraba en proceso de colonización

Volumen maderable, fuste limpio y área basal

El volumen total estimado de madera fue de $68.5 \mathrm{~m}^{3} \mathrm{ha}^{-1}$, siendo el mangle negro el que presentó el mayor volumen con $68.3 \mathrm{~m}^{3} \mathrm{ha}^{-1}$., siendo $38.3 \mathrm{~m} 3$ de árboles totalmente muertos y el restante $30.0 \mathrm{~m}^{3} \mathrm{ha}^{-1}$ a árboles parcialmente vivos. De esta manera el volumen total maderable fue de $3,425 \mathrm{~m}^{3}$ para las 50 hectáreas de manglar evaluadas.

El volumen maderable del fuste limpio afectado (Vfl) promedio del mangle negro fue de $24.6 \mathrm{~m}^{3} \mathrm{ha}^{-1}$, las unidades de monitoreo 1 y 9 , registraron el menor y mayor volumen maderable con 9.1 y $61.0 \mathrm{~m} 3 \mathrm{ha}-1$ respectivamente, dependiendo si el sitio afectado correspondió a arboles en desarrollo o arboles adultos. El Vfl promedio de árboles vivos fue de $11.5 \mathrm{~m}^{3} \mathrm{ha}^{-1} \mathrm{y}$ para árboles muertos $13.1 \mathrm{~m}^{3} \mathrm{ha}^{-1}$.

El área basal $(\mathrm{AB})$ total fue de $12.4 \mathrm{~m}^{2} \mathrm{ha}^{-1}$; de los cuales 4.9 correspondieron a árboles parcialmente vivos y el restante $7.6 \mathrm{~m}^{2} \mathrm{ha}^{-1}$ a árboles muertos; respectivamente. $\mathrm{El} \mathrm{AB}$ promedio del mangle negro (A.germinans) fue de $12.4 \mathrm{~m} 2 \mathrm{ha}^{-1}$; 4.8 y $7.5 \mathrm{~m}^{2} \mathrm{ha}^{-1}$ de árboles vivos y muertos; respectivamente. Esta disparidad en los datos se debe a la heterogeneidad en la estructura del manglar, pues se registraron sitios con alto porcentaje de arboles juveniles, mientras que otros eran dominados por arboles adultos y otros con valores intermedios (Yañez et al., 2009).

\section{Clase diamétrica}

Los valores en DAP variaron de 0.3 a $36 \mathrm{~cm}$, con una media de $8.2 \mathrm{~cm}$. Del total de los árboles evaluados la clase diamétrica de $5-9.9 \mathrm{~cm}$ de DAP registró el mayor número de árboles con 821 (45.0\%), seguido por la clase $0.1-4.9 \mathrm{~cm}$ con 451 árboles $(24.7 \%)$. Las primeras tres clases diamétricas $(1-14.9 \mathrm{~cm})$ concentraron el 92.1\% del total (Figura 2); de acuerdo a su tasa de crecimiento estos árboles se encuentran en etapa juvenil o en fase temprana reproductiva, lo que indica que en ese rango es susceptible a ataque y defoliación por la oruga. Esto indica que los individuos que resultaron afectados pertenecen a una población relativamente joven dado que esta especie puede tener diámetros por arriba de los $100 \mathrm{~cm}$ en condiciones óptimas de desarrollo y en edad adulta. 


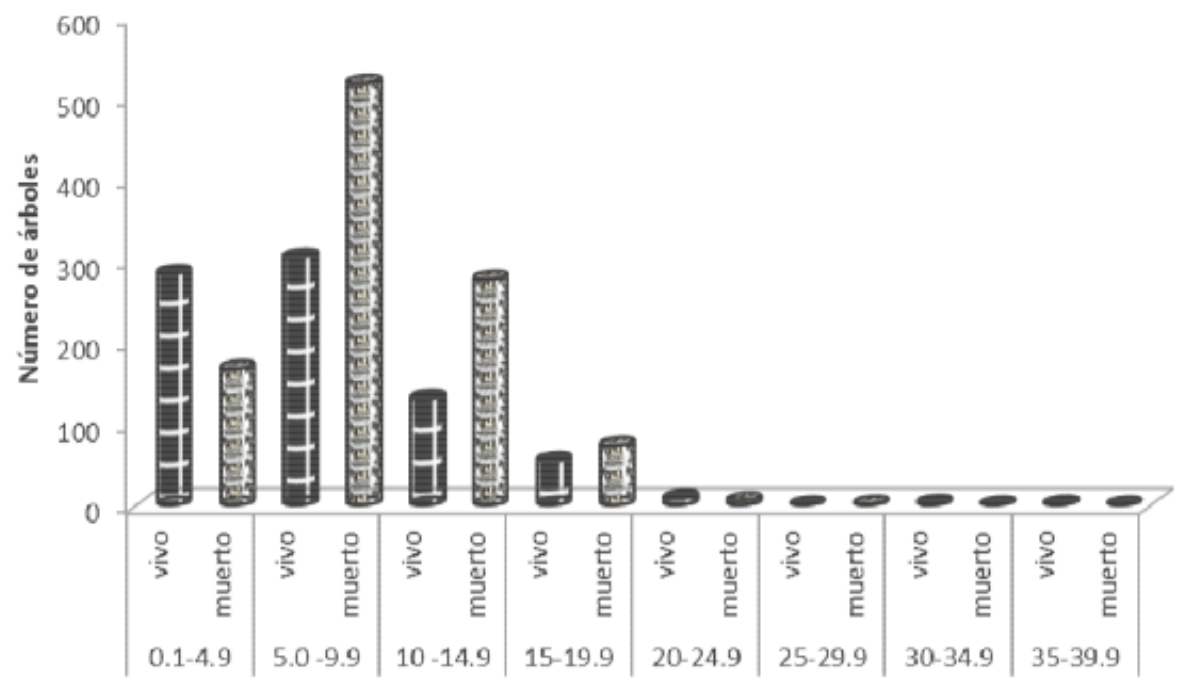

FIGURA 2

Distribución de los árboles por clase diamétrica en el ecosistema manglar afectado en Cárdenas, Tabasco, México.

Elaboración propia.

\section{Análisis de correlación simple entre el DAP y la altura total del mangle negro}

La correlación que guardaron el DAP y la altura del mangle negro, indican una moderada relación positiva $\left(\mathrm{r}^{2}=0.5313, \mathrm{p}=2.2 \mathrm{e}^{-16}\right)$ (Figura 3 ), esta condición puede atribuirse por el ataque de la oruga de Anacamptodes sp, donde alrededor del 60\% de manglar empleado en esta correlación correspondió a mangle muerto por defoliación, árboles quebrados y desraizados. La media del DAP y la altura fueron de $8.2 \mathrm{~cm}$ y $5.9 \mathrm{~m}$ respectivamente. Esto indica que la plaga prefiere árboles jóvenes por su posibilidad de ovipositar en ellos, además de que esta altura se encuentra dentro del rango de vuelo del adulto de la plaga. 


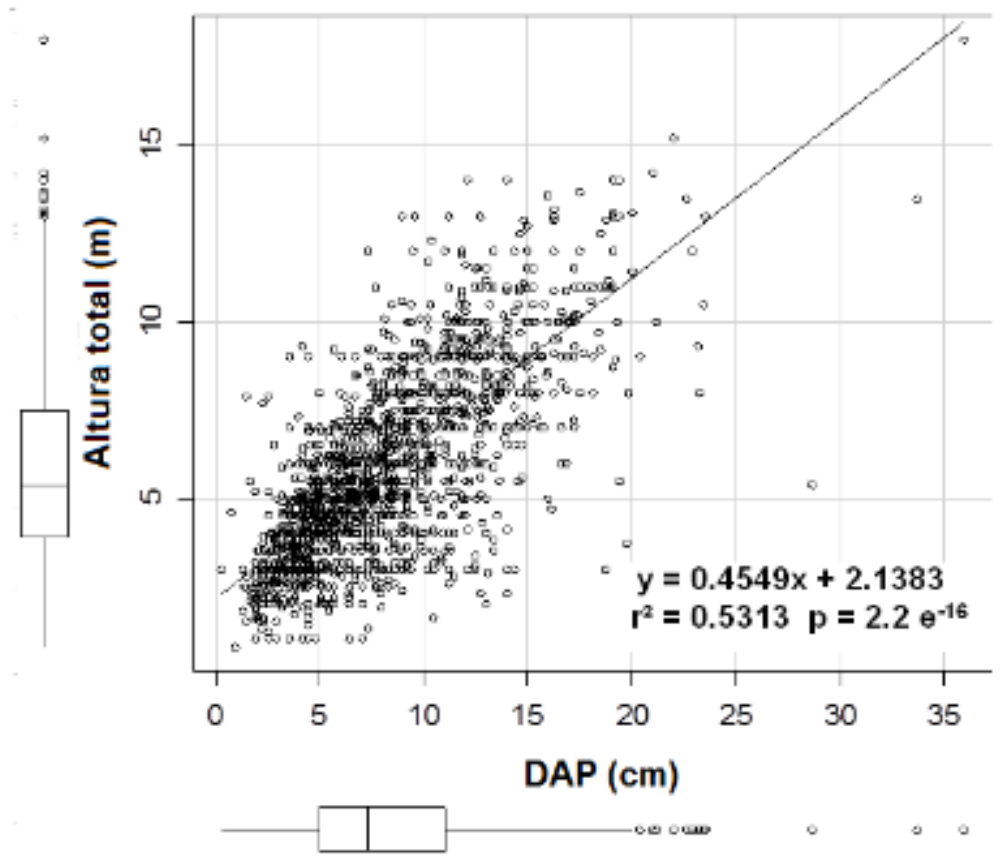

FIGURA 3

Correlación entre el diámetro a la altura de pecho $(\mathrm{DAP} \mathrm{cm}) \mathrm{y}$ la altura $(\mathrm{m})$ del total de los árboles de mangle negro registrados. Elaboración propia

\section{Discusión}

Las unidades de muestreo estuvieron mayormente representadas por árboles de mangle negro, con diámetros promedio de $8 \mathrm{~cm}$, que por su apariencia forestal corresponden a una zona en proceso de colonización. Los árboles presentes fueron jóvenes con alta susceptibilidad al ataque de plagas, razón por la cual el 56\% de los árboles evaluados se encontraban en estado seco o muerto.

La altura promedio de los árboles fue de $5.9 \mathrm{~m}$; sin embargo, Tovilla et al. (2009) en Chetumal, Quintana Roo registraron una media de $8.3 \mathrm{~m}$. y Basáñez et al. (2008) en Tuxpan, Veracruz registraron altura promedio de $3.1 \mathrm{~m}$. La altura depende de variables como las condiciones edafoclimáticas de la zona y la edad de la planta. En el caso de la Comunidad de Las Coloradas, las poblaciones evaluadas corresponden a árboles juveniles.

En Costa Rica, Fonseca et al. (2007) registraron árboles maduros y seniles de mangle con una altura promedio de $10.7 \mathrm{~m}$. Asimismo, en una población sana de árboles de mangle en Turrialba costa rica Ammour et al. (1999) registraron alturas promedios de $18 \mathrm{~m}$., condición que se le atribuye a las características propias del suelo.

La densidad de árboles en el sitio estudiado es similar a la densidad registrada para manglares de otros sitios de México y del mundo; para Chetumal Tovilla et al. (2009) reportan 1,886 árboles ha-1; para Nayarit Tovilla y reportan 1,994 árboles ha-1. En similar condición; Ammour et al. (1999) reportan una densidad de 1,600 a 2,200 árboles ha ${ }^{-1}$ para Costa Rica.

En relación al volumen maderable, los datos registrados en el presente estudio, presentan diferencias con trabajos similares de otras regiones del mundo. La cual depende de directamente de la edad del ecosistema y del número de individuos por unidad de área presente. Así Ammour et al. (1999), Fonseca et al. (2007) en Costa Rica registraron de 20 a $50 \mathrm{~m}^{3} \mathrm{ha}^{-1}$, volumen menor que el resultado registrado en las mediciones de este trabajo. Estas mismas variables califican para el diámetro de los árboles evaluados. 
El área basal promedio del arbolado evaluado fue de $12.4 \mathrm{~m}^{2} \mathrm{ha}^{-1}$, variando de $5.4 \mathrm{~m}_{2} \mathrm{ha}^{-1}$ a $25 \mathrm{~m}^{2} \mathrm{ha}^{-1}$, similar a los resultados de Fonseca et al, (2007) en Costa Rica, pero diferentes a los de Basáñez et al. (2008) en Tuxpan y Tovilla et al. (2009) en Quintana Roo La correlación que guardan el DAP y la altura fue moderada relación positiva $\left(\mathrm{r}^{2}=0.5313, \mathrm{p}=2.2 \mathrm{e}-16\right)$. Tovilla et al. (2009) en Quintana Roo registraron una alta relación $\left(\mathrm{r}^{2}\right.$ $=0.88$ ), mayor que nuestro estudio, condición que puede atribuirse a la edad del arbolado evaluado, pues en su mayoría este se concentró en plantas en juveniles. Otra variable que influye es el estado de salud del ecosistema.

\section{CoNCLUSIón}

El impacto del daño sobre las poblaciones de mangle fue severo, en virtud de que el sitio donde estuvo presente la oruga de Anacamptodes quedo despojado de cualquier tipo de vegetación, por lo que la recuperación natural podría darse de manera gradual, colocando en riesgo las especies acuáticas y terrestres que ahí se reproducen, y que representan la fuente de ingresos económicos de las comunidades aledañas, por lo que es necesario reforestar de manera urgente.

La altura de vuelo del adulto de Anacamptodes favoreció a los arboles adultos porque se encuentran fuera de rango de vuelo de la misma, pero ubico como más vulnerable a las plantas en desarrollo, pues se ubican dentro de las posibles alturas de vuelo, por esta razón hubo mayor número de plantas defoliadas y muertas en la clase diamétrica $5-14.9 \mathrm{~cm}$

Es necesario ayudar al ecosistema con actividades de restauración, dado que en la actualidad la regeneración natural no ha sido exitosa en virtud de que los árboles que se mantienen vivos no han producido semillas. Es conveniente dar seguimiento a la especie de Anacamptodes sp, aunque su presencia actual es tolerable pues no representa mayor peligro en relación al año de la defoliación. En la actualidad la población de esta especie se encuentra por debajo del umbral en que podría causar algún daño

\section{Agradecimientos}

A la Comisión Nacional Forestal, por el apoyo brindado para el desarrollo de este estudio. A la línea Prioritaria de Investigación 8 del Colegio de Postgraduados "Impacto y Mitigación del Cambio Climático", por las facilidades prestadas para el desarrollo del presente trabajo.

\section{BIBLIOGRAFÍA}

Andenmatten, E. y Letourneau F. (2000). Estimación de alturas para su empleo en tablas de volumen de árbol individual. Comunicación técnica núm. 10 área forestal silvicultura. Instituto Nacional de Tecnología Agropecuaria (INTA). 8 p.

Ammour, T., Imbach A., Suman D. y Windevoxhel L. N. (1999). Manejo productivo de Manglares en América Central. (7). CATIE.

Basáñez A. J., Cruz M. A., Domínguez B. C., González G. C., Serrano S. A. y Hernández A. A. (2008). Estructura y producción de Conocarpus erectus L. en el Sitio Ramsar "Manglares y Humedales de Tuxpan", Veracruz, México. UDO Agrícola, 8 (1): 78-87.

Berlanga, R. C. A. y Ruiz L. A. (2007). Análisis de las tendencias de cambio del bosque de mangle del sistema lagunar Teacapán - Agua Brava, México. Una aproximación con el uso de imagines de satélite Landsat. Universidad Juárez Autónoma de Tabasco. Pp. 29 - 46.

Castillo, B. E., Gervasio J. H., Martínez J. L. y Rosas J. L. (2010). Estructura y composición florística de una zona de manglar anexa con las localidades de Playa Azul y El Carrizal, Municipio de Coyuca de Benítez, Guerrero. 
Memoria de XV Foro de estudios sobre Guerrero, Acapulco, Guerrero, México. 26 de noviembre del 2010. CDROM.

CONABIO. (2008). Manglares de México. Comisión Nacional para el Conocimiento y Uso de la Biodiversidad. Tlalpan, México, D.F. 39 p.

Díaz J. M. (2011). Una revisión sobre los manglares: características, problemáticas y su marco jurídico. Importancia de los manglares, el daño de los efectos antropogénicos y su marco jurídico: caso sistema lagunar de topolobampo. Ra Ximhai, 7, (3): 355-369.

Flores-Verdugo, F.J. Agraz-Hernández C. y Benítez-Pardo D. (2007). Ecosistemas acuáticos costeros: importancia, retos y prioridades para su conservación. In: Sánchez O., Herzig M., Peters E., Márquez R., y Zambrano L. Perspectivas sobre conservación de ecosistemas acuáticos en México. 1era. Edición.

Fonseca A. C., Cortés J. y Zamora P. (2007). Monitoreo del manglar de Gandoca, Costa Rica (sitio CARICOMP). Rev. Biol. Trop. 55 (1): 23-31.

Foroughbakhch, P. R., Céspedes A. E., Alvarado M. A., Núñez G. A. y Badii M. H. (2004). Aspectos ecológicos de los manglares y su potencial como fitorremediadores en el Golfo de México. Ciencia. Universidad Autónoma de Nuevo León, Monterrey, México. 7 (2): 203-208.

Gallegos, M., y Botello A. V. (1988). Petróleo y manglar. Proyecto General: Evaluación de los Impactos Ambientales y Sociales de la Industria Petrolera en el Sureste y Golfo de México. Centro de Ecodesarrollo. Primera Edición.

García, L. E. Zavala C., J. y Palma D.J. (2006). Caracterización de las comunidades vegetales en un área afectada por derrames de hidrocarburos. Terra Latinoamericana 34:17-26.

Góngora, R. F., Moreno A. R. y Quintana P. X. (2004). Número de árboles por hectárea como variable predictora de la cantidad de huevos de Ochlerotatus taeniorhynchus wiedemann (Diptera: culicidae) en manglares al sur de Pinar del Río. Revista Forestal Baracoa. 23 (2):47-54.

Góngora, R. F. (2005). Usos de número de árboles por hectárea en el manglar para predecir los sitios de oviposición de los mosquitos costeros. Serie ciencias forestales y del ambiente. 11 (1): 19-23.

Hernández J. M., Ortiz M. A., Méndez A. P., y Gama L. (2008). Morfo dinámica de la línea de costa del estado de Tabasco, México: tendencias desde la segunda mitad del siglo XX hasta el presente. Investigaciones Geográficas, Boletín del Instituto de Geografía, UNAM. Núm. 65. México. Pp.

Hernández G. I., Sol S. A., Ruiz R. O., Valdez J. I. López C. J. (2013) Legislación, cambio de uso de suelo, y reforestación en manglares de Cárdenas, Tabasco. Tesis de doctorado en ciencias. Colegio de Postgraduados. Campus Veracruz

INE y SEMARNAT. 2005. Evaluación preliminar de las tasas de pérdida de superficie de manglar en México. 21 p.

Moreno, C. E., Guerrero P. A., Gutiérrez C. M. C., Ortiz S. C. A y Palma L. D. J. (2002). Los manglares de Tabasco, una reserva natural de carbono. Madera y Bosques, (Número especial): 115-128.

Olguín, E. J. Hernández M. E. y Sánchez G. G. (2007). Contaminación de manglares por hidrocarburos y estrategias de biorremediación, fitorremediación y restauración. Revista Internacional de Contaminación Ambiental. 23: 139-154.

Sol S. A., Zenteno C. L., Zamora L. F., Torres R. E. (2002). Modelo para la Restauración Ecológica en áreas alteradas. Revista de difusión Kuxulcab, 7(14) 48-60.

Sol S. A., Hernández G.I. Sánchez G. F. (2012). Volumen de madera de mangle negro (Avicennia germinans L.) muerto defoliado por orugas de Anacamptodes sp en el ejido las coloradas, Cárdenas Tabasco. México. pp 33 -34 en: segundo congreso México sobre el ecosistema de manglar. 2226 octubr2012. Cd del Carmen Campeche.

Tovilla-Hernández, C., Orihuela-Belmonte, D. E., \& Salas-Robledo, L. (2009). Estructura, composición, regeneración y extracción de madera de los bosques de manglar. El sistema ecológico de la bahía de Chetumal/Corozal: costa occidental del mar Caribe, J. Espinoza-Ávalos, GA Islebe y HA Hernández-Arana (eds.). El Colegio de la Frontera Sur, Chetumal, 45-60.

Tovilla, H. C. y Orihuela D. E. (2004). Impacto del huracán Rosa sobre los bosques de manglar de la costa norte de Nayarit, México. Madera y Bosques, 10 (2): 63-75. 
Revista Iberoamericana de Bioeconomía y Cambio Climático, 2015, vol. 1, núm. 1, Enero-Julio, ISSN...

Rico, G. V. (1982). Rhizophora harrisonii (Rhizophoraceae), un nuevo registro de las costas de México. Boletín de la Sociedad Botánica de México 41: 163165.

Valdez J. I. (2004). Manejo forestal de un manglar al sur de marismas nacionales, Nayarit. Madera y Bosques. 10, (2) 93-104.

Valdez J. I. (2002). Aprovechamiento forestal de manglares en el estado de Nayarit, costa Pacífica de México. Madera y Bosques. 8 (1): 128-145.

Yáñez, E. L., Ángeles G., López P. J. y Barrales S. (2009). Variación anatómica de la madera de Avicennia germinans en la Laguna de la Mancha, Veracruz, México. Bol. Soc. Bot. Méx. 85: 7-15 